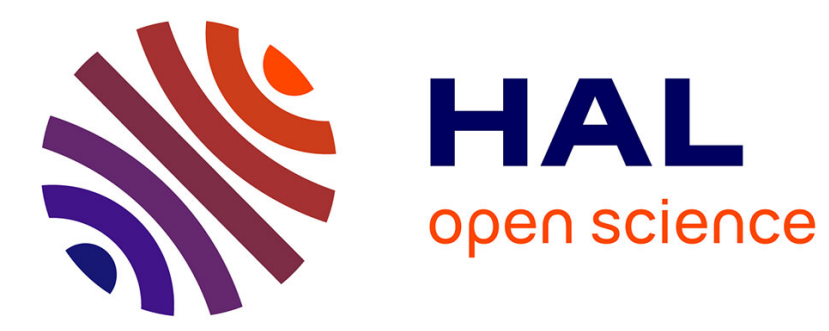

\title{
Energy-Efficient Relay Assignment and Power Control in Multi-User and Multi-Relay Networks
}

\author{
A. Zappone, S. Atapattu, M. Di Renzo, J. Evans, M. Debbah
}

\section{To cite this version:}

A. Zappone, S. Atapattu, M. Di Renzo, J. Evans, M. Debbah. Energy-Efficient Relay Assignment and Power Control in Multi-User and Multi-Relay Networks. IEEE Wireless Communications Letters, 2018, pp.1 - 1. 10.1109/LWC.2018.2860013 . hal-01880309

\section{HAL Id: hal-01880309 \\ https://hal.science/hal-01880309}

Submitted on 18 Jun 2020

HAL is a multi-disciplinary open access archive for the deposit and dissemination of scientific research documents, whether they are published or not. The documents may come from teaching and research institutions in France or abroad, or from public or private research centers.
L'archive ouverte pluridisciplinaire HAL, est destinée au dépôt et à la diffusion de documents scientifiques de niveau recherche, publiés ou non, émanant des établissements d'enseignement et de recherche français ou étrangers, des laboratoires publics ou privés. 


\title{
Energy-Efficient Relay Assignment and Power Control in Multi-User and Multi-Relay Networks
}

\author{
A. Zappone, Senior Member, IEEE, S. Atapattu, Member, IEEE, M. Di. Renzo, Senior Member, IEEE, J. Evans, \\ Senior Member, IEEE, and M. Debbah, Fellow, IEEE
}

\begin{abstract}
We consider a multi-user, multi-relay network where each source communicates with its destination via an assigned relay. To ensure fairness among the users, the minimum of the users' energy efficiencies (in bit-per-Joule) is jointly maximized with respect to the relay assignment and transmit power of both source and relay nodes. The resulting algorithm is provably convergent, requires limited complexity, and significantly outperforms the baseline scenario in which no joint optimization of transmit powers and relay assignment is performed.
\end{abstract}

Index Terms-Energy efficiency, multi-user networks, multirelay networks, relay assignment, power optimization.

\section{INTRODUCTION}

Radio resource allocation for energy efficiency has become a key performance indicator for the design of future mobile networks [1]. Furthermore, the use of relays constitutes a fundamental tool for extending the coverage of current and future wireless networks [2]. In the context of single- or multiuser networks, where multiple relays are available, properly designed relay assignment strategies have the potential of providing spatial diversity gains to the users, thus improving their performance [3]. The relay assignment problem is usually formulated to improve the error rate and throughput of the source-to-destination links, by assuming that their transmit powers are fixed, e.g. [4], [5]. The rise of energy efficiency as a key performance indicator for wireless networks, however, has put forth the relevance of investigating the relay assignment problem from an energy efficiency perspective.

The design of relay assignment schemes that aim at optimizing the energy efficiency is considered in several recent works focused on single-user networks [6]-[10] (and references therein). The goal is to minimize the transmit power of a multi-user orthogonal frequency-division multiplexing system. In [11], a power-allocation method that reduces the total power consumption while maintaining the required quality of service for application to single-user and multi-user relay systems is proposed. Other recent works study power allocation schemes for relay-aided networks, considering a fixed assignment. In

A. Zappone, M. Di Renzo, M. Debbah are with CentraleSupeléc-CNRSUniv. Paris Sud, France (e-mail: \{alessio zappone, marco.direnzo, merouane.debbah\}@12s.centralesupelec.fr). M. Debbah is also with the Mathematical and Algorithmic Sciences Lab, Huawei France R\&D, Paris, France (merouane.debbah@huawei.com). S. Atapattu and J. Evans are with the Department of Electrical and Electronic Engineering, The University of Melbourne, Melbourne, Australia (e-mail: \{saman.atapattu,jse\}@unimelb.edu.au).

The work of A. Zappone and M. Debbah was supported by the EC through the H2020-MSCA IF-BESMART project (grant 749336) and the H2020ERC PoC-CacheMire project (grant 727682). The work of M. Di Renzo was supported by the EC through the H2020-MSCA ETN-5Gwireless project (grant 641985).

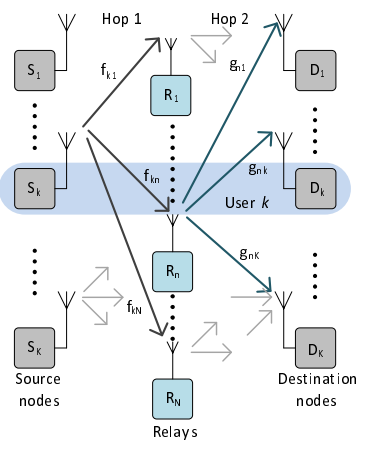

Fig. 1: Considered multi-user multi-relay network model.

[12], the energy efficiency of a multi-user and single-relay MIMO system is maximized. In [13], a relay-assisted cognitive system is considered. An information-theoretic analysis of the multi-way relay channel is available in [14]. Energyefficient resource allocation policies for the multi-way relay channel are studied in [15]. In [16], schemes that minimize the energy expenditure of cloud radio relay-assisted networks under quality of service constraints are analyzed.

In the depicted context, the present work considers a multiuser and multi-relay network, and aims at jointly optimizing the relay assignment and transmit powers to maximize the minimum bit-per-Joule energy efficiency across the users, a problem that has not been considered in previous works. Indeed, as outlined above, most previous works focus only on either relay assignment or power control. Moreover, available works that jointly consider relay assignment and power control are focused on non-energy-efficient allocations, like [17], where a joint optimization of relay selection and resource allocation under rate constraints is considered.

The novel scenario considered in this work leads to a mixedinteger optimization problem, which is tackled by generalizing to the energy-efficient scenario the relay assignment strategies from [5], where SNR maximization was considered, and by employing generalized fractional programming tools to deal with the power allocation part of the problem [1].

\section{System ModeL}

We consider the dual-hop wireless relay network depicted in Fig. 1, where $K$ source nodes $\left(S_{1}, \ldots, S_{K}\right)$ send information to their corresponding destination nodes $\left(D_{1}, \ldots, D_{K}\right)$ via $N$ relay nodes $\left(R_{1}, \ldots, R_{N}\right)$ that operate either in decode-andforward (DF) or in amplify-and-forward (AF) mode. Each node is equipped with a single antenna and operates in halfduplex mode. The transmit powers of the $k$ th user and $n$th 
relay, $n \in\{1, \ldots, N\}$, are $p_{k}$ and $q_{n}$, respectively. Motivated by practical considerations, we consider the case in which each user is assisted by one and only one relay ${ }^{1}$, thus assuming $N \geq K$. To avoid interference among the users, orthogonal (time, frequency) channels are used for transmission.

The fading channel between $S_{k}$ and $R_{n}$ and between $R_{n}$ and $D_{k}$ are denoted by $f_{k, n}$ and $g_{k, n}$, respectively. All channels are assumed to be independent and identically distributed, to remain constant during one transmission block, but to change independently over different transmission blocks. Moreover, the direct user-destination channels are assumed to be too weak for communication and hence are neglected. Then, the achievable rate, $r_{k, n}$, and bit-per-Joule energy efficiency, $\eta_{k, n}$, of the $k$ th user-destination pair are:

$$
\begin{aligned}
r_{k, n} & =\frac{1}{2 K} \log _{2}\left(1+\gamma_{k, n}\left(p_{k}, q_{n}\right)\right)[\mathrm{bpcu}] \\
\eta_{k, n} & =\frac{B \log _{2}\left(1+\gamma_{k, n}\left(p_{k}, q_{n}\right)\right)}{\lambda_{k} p_{k}+\mu_{n} q_{n}+P_{k, n}^{(c)}} \text { [bit/Joule] }
\end{aligned}
$$

where "bpcu" stands for bit per channel use, the pre-log factor $1 / 2 K$ is due to the half-duplex operation mode and to the use of orthogonal transmission channels, $\lambda_{k}$ and $\mu_{n}$ are the inverse of the power amplifier efficiencies of $S_{k}$ and $R_{n}$, respectively, $P_{k, n}^{(c)}$ is the static power dissipated to operate the communication of user $k$ through relay $n$, and $\gamma_{k, n}$ is the SNR of the $S_{k}-D_{k}$ pair through relay $R_{n}$, which depends on the particular relaying protocol being used. Let $\gamma_{k, n}^{(1)}=p_{k} \frac{\left|f_{k, n}\right|^{2}}{\sigma^{2}}$ and $\gamma_{k, n}^{(2)}=q_{n} \frac{\left|g_{k, n}\right|^{2}}{\sigma^{2}}$, be the SNRs of the $S_{k}-R_{n}$ and $R_{n}-D_{k}$ links, where $\sigma^{2}$ is the noise power. The SNRs enjoyed using the DF and AF relaying protocols are, respectively:

$$
\begin{gathered}
\gamma_{k, n}^{D F}\left(p_{k}, q_{n}\right)=\min \left(\gamma_{k, n}^{(1)}, \gamma_{k, n}^{(2)}\right)=\frac{\min \left(p_{k}\left|f_{k, n}\right|^{2}, q_{n}\left|g_{k, n}\right|^{2}\right)}{\sigma^{2}} \\
\gamma_{k, n}^{A F}\left(p_{k}, q_{n}\right)=\frac{\gamma_{k, n}^{(1)} \gamma_{k, n}^{(2)}}{\gamma_{k, n}^{(1)}+\gamma_{k, n}^{(2)}+1}=\frac{p_{k} q_{n}\left|f_{k, n}\right|^{2}\left|g_{k, n}\right|^{2} / \sigma^{2}}{p_{k}\left|f_{k, n}\right|^{2}+q_{n}\left|g_{k, n}\right|^{2}+\sigma^{2}} .
\end{gathered}
$$

Motivated by fairness considerations ${ }^{2}$, our goal is to maximize the minimum energy efficiency across the users, with respect to $\left\{p_{k}\right\}_{k=1}^{K},\left\{q_{n}\right\}_{n=1}^{N}$, and to the source-relay association $\left\{\delta_{k, n}\right\}_{k, n}$, where $\delta_{k, n}=1$ if user $k$ is assisted by relay $n$, and $\delta_{k, n}=0$ otherwise.

\section{Problem Formulation AND SOlution}

The energy efficiency of the $k$ th user before knowing its associated relay is given by ${ }^{3}$ :

$$
\eta_{k}=\frac{\sum_{n=1}^{N} \log _{2}\left(1+\delta_{k, n} \gamma_{k, n}\left(p_{k}, q_{n}\right)\right)}{\lambda_{k} p_{k}+\sum_{n=1}^{N} \delta_{k, n}\left(\mu_{n} q_{n}+P_{k, n}^{(c)}\right)} .
$$

Then, the maximization of the minimum energy efficiency among the users can be formulated as follows:

$$
\begin{aligned}
& \max _{\left\{p_{k}, q_{n}, \delta_{k, n}\right\}_{k, n}} \min _{1 \leq k \leq K} \eta_{k}\left(p_{k}, q_{n}, \delta_{k, n}\right) \\
& \text { s.t. } p_{k} \in\left[0, p_{\text {max }, k}\right], q_{n} \in\left[0, q_{\text {max }, n}\right] \forall k, n \\
& \delta_{k, n} \in\{0,1\}, \sum_{n=1}^{N} \delta_{k, n}=1, \sum_{k=1}^{K} \delta_{k, n} \in\{0,1\} \forall k, n
\end{aligned}
$$

\footnotetext{
${ }^{1}$ Having multiple users associated to multiple relays would increase the system complexity and energy consumption [18], [19].

${ }^{2}$ If instead a system-wide performance optimization is desired, the system global energy efficiency is a more suitable performance metric.

${ }^{3}$ Recall that the users are assigned to only one relay.
}

where $p_{\max , k}$ and $q_{\max , n}$ are the maximum power levels of $S_{k}$ and $R_{n}$, respectively, while (6c) ensures that each user is connected to one and only one relay.

The main challenges to solve (6) lie in its mixed-integer nature and in its non-concave fractional objective, which is not differentiable even with respect to only the transmit powers due to the $\min (\cdot)$ operator. We tackle these issues by using the alternating optimization method illustrated in Algorithm 1 , alternatively optimizing the association variables $\left\{\delta_{k, n}\right\}_{k, n}$ for fixed transmit powers $\left\{p_{k}, q_{n}\right\}_{k, n}$, and the transmit powers for fixed association variables, until convergence.

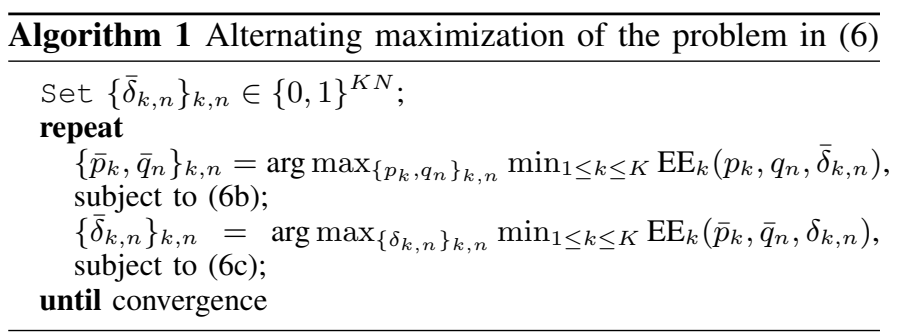

Algorithm 1 monotonically increases the value of the objective and converges in the value of the objective ${ }^{4}$, even though global optimality cannot be theoretically guaranteed. The two optimization subproblems that constitute Algorithm 1 are discussed and solved in the next two sections.

\section{A. Transmit Power Optimization}

The first subproblem in Algorithm 1 assumes that the association variables $\delta_{k, n}=\bar{\delta}_{k, n}$ are fixed for all $k, n$, and optimizes the energy efficiency with respect to $\left\{p_{k}\right\}_{k}$ and $\left\{q_{n}\right\}_{n}$. Thus, the following optimization problem is considered:

$$
\begin{aligned}
& \max _{\left\{p_{k}, q_{n}\right\}_{k, n}} \min _{1 \leq k \leq K} \eta_{k}\left(p_{k}, q_{n}\right) \\
& \text { s.t. } p_{k} \in\left[0, p_{\max , k}\right],\left[0, q_{\max , n}\right] \forall k, n .
\end{aligned}
$$

The optimization problem in (7) is an instance of a generalized fractional program [20], which differs from the well-known class of fractional programs because of the presence of the $\min (\cdot)$ operator. An algorithm to globally solve generalized fractional programs was proposed in [20, Section 2.2] and named generalized Dinkelbach's algorithm. If each ratio within the $\min (\cdot)$ operator is the ratio of a concave function over a convex function, and all the constraints are convex, then the generalized Dinkelbach's algorithm finds the solution of (7) by solving a sequence of convex auxiliary problems that converge at a rate that is at least linear [20, Proposition 3.3]. As far as the specific problem at hand is concerned, the denominators in (7a) and the affine constraints in (7b) fulfill these requirements. Instead, as for the numerators in (7a), the analysis is more challenging. In particular, the DF and AF protocols need to be treated separately.

Proposition 3.1: Let the DF protocol be used. Every numerator in (7a) is jointly concave in $p_{k}$ and $q_{n}$, for all $k, n$.

Proof: It needs to be proved that $\gamma_{k, n}$ is jointly concave in $p_{k}$ and $q_{n}$, for all $k, n$. This follows since the logarithm of a concave function is concave and the sum of concave

\footnotetext{
${ }^{4}$ The objective (6a) is upper-bounded over the feasible set and thus it cannot increase indefinitely.
} 
functions is concave. Also, $\gamma_{k, n}$ in (3) is the minimum of linear functions and, thus, it is concave, since the minimum of concave functions is concave.

Proposition 3.2: With the AF protocol, every numerator in (7a) is jointly concave in $p_{k}, q_{n}$ if $\gamma_{k, n}^{(1)} \gamma_{k, n}^{(2)} \geq \frac{1}{2}$ for all $k, n$.

Proof: Similar to DF, it needs to be proved that $\gamma_{k, n}$ is jointly concave in $p_{k}$ and $q_{n}$, for all $k, n$. We observe that (4) can be equivalently studied as a function of $\gamma_{k, n}^{(1)}$ and $\gamma_{k, n}^{(2)}$, or as a function of $p_{k}$ and $q_{n}$, since the two pairs of variables are related by a linear mapping that preserves concavity. We study the concavity of (4) as a function of $\gamma_{k, n}^{(1)}$ and $\gamma_{k, n}^{(2)}$, by computing its Hessian matrix. With some algebra, we obtain (8) shown at the top of the next page. The diagonal elements of (8) are non-positive, and, thus, it is negative semi-definite if its determinant is non-negative ${ }^{5}$. This is equivalent to:

$$
\begin{aligned}
& 2 p_{k} q_{n}\left(p_{k}^{2}+2 p_{k}+q_{n}^{2}+2 q_{n}+2 p_{k} q_{n}\right)-\left(p_{k}^{2}+2 p_{k}+q_{n}^{2}+2 q_{n}+1\right) \\
& =\left(2 p_{k} q_{n}-1\right)\left(p_{k}^{2}+2 p_{k}+q_{n}^{2}+2 q_{n}+2 p_{k} q_{n}+1\right) \geq 0
\end{aligned}
$$

from which the proof follows.

Remark 1: The condition $\gamma_{k, n}^{(1)} \gamma_{k, n}^{(2)} \geq \frac{1}{2}$ in Proposition 3.2 is not very restrictive. The term $\gamma_{k, n}^{(1)} \gamma_{k, n}^{(2)}$, in fact, is the product of the SNRs at the relay and destination, which cannot be too low to ensure a reliable communication link.

\section{B. Users-Relay Association Optimization}

The second subproblem in Algorithm 1 assumes that the transmit powers $p_{k}=\bar{p}_{k}$ and $q_{n}=\bar{q}_{n}$ are fixed for all $k, n$, and optimizes the energy efficiency with respect to $\left\{\delta_{k, n}\right\}_{k, n}$. Thus, the following optimization problem is considered:

$$
\begin{aligned}
& \max _{\left\{\delta_{k, n}\right\}_{k, n}} \min _{1 \leq k \leq K} \eta_{k}\left(\bar{p}_{k}, \bar{q}_{n}, \delta_{k, n}\right) \\
& \text { s.t. } \delta_{k, n} \in\{0,1\}, \sum_{n=1}^{N} \delta_{k, n}=1, \sum_{k=1}^{K} \delta_{k, n} \in\{0,1\} \forall k, n .
\end{aligned}
$$

The optimal solution of (10) can be found as follows. Since the transmit powers $\left\{\bar{p}_{k}\right\}_{k=1}^{K}$ and $\left\{\bar{q}_{n}\right\}_{n=1}^{N}$ are given, one can stack, for any possible relay assignment, the energy efficiencies of all the users in a $K \times N$ matrix $\mathcal{E}=\left\{\eta_{k, n}\right\}_{k, n}$, whose elements are $\eta_{k, n}=\frac{B \log _{2}\left(1+\gamma_{k, n}\left(\bar{p}_{k}, \bar{q}_{n}\right)\right)}{\lambda_{k} \bar{p}_{k}+\mu_{n} \bar{q}_{n}+P_{k, n}^{(c)}}$ (see (2)). The $k$ th row gives the energy efficiencies of the $k$ if assisted by relay $\left\{R_{n}\right\}_{n=1}^{N}$, and the $n$th column gives the energy efficiencies of all the users if assisted by Relay $R_{n}$. Since a given relay can assist the transmission of only one user, a selection policy is admissible if only one entry of each row is non-zero, and if each of those entries is in a different column. Since the max-min problem in (10) considers individual performance and fairness, an optimal assignment can be obtained by generalizing the algorithms from [4], [5], to the energy efficiency maximization scenario.

In particular, we propose the following two-step algorithm.

Step I: The energy efficiency matrix $\mathcal{E}$ is computed as explained above. Algorithm 2 is applied to $\mathcal{E}$ to maximize the minimum energy efficiency across the users. Let $u$ and $R_{u}$ be the user and the associated relay that achieve the optimum.

\footnotetext{
${ }^{5}$ Recall that a $2 \times 2$ Hermitian matrix is non-negative semidefinite if its $(1,1)$-element is negative and if its determinant is non-negative.
}

Step II: After Step I, the $u$ th row and $R_{u}$ th column of $\mathcal{E}$ are deleted and Algorithm 2 is applied to the new matrix $\mathcal{E}_{u}$. This is iterated until all users are associated to a relay.

Both steps use Algorithm 2 to operate on a given energy efficiency matrix by the functions ${ }^{6}$ find_another_relay () and check_relay_availability(), where the user associated to relay $R_{j}$ is denoted by $\mathcal{U}\left(R_{j}\right)$ and its energy efficiency by $\eta\left(\mathcal{U}\left(R_{j}\right), R_{j}\right)$.

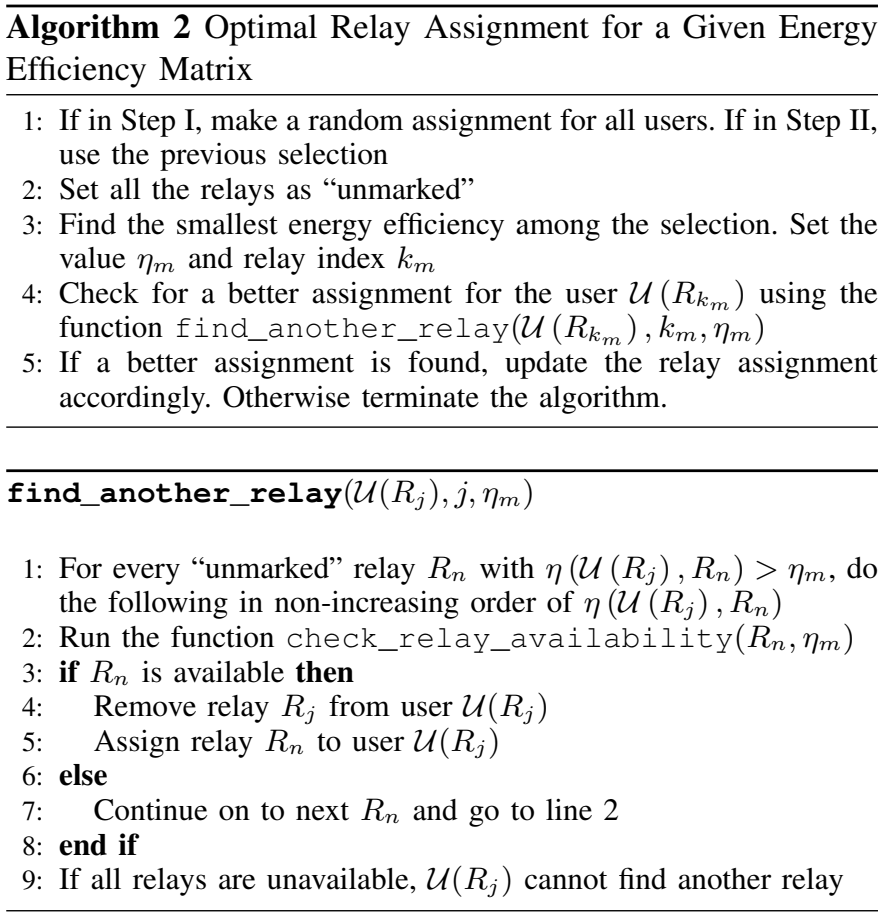

check_relay_availability $\left(R_{j}, \eta_{m}\right)$

1: if $R_{j}$ is not assigned to any user or $R_{j}=R_{k_{m}}$ then

2: $\quad R_{j}$ is available.

3: else

4: Set $R_{j}$ as "marked"

5: $\quad$ Run find_another_relay $\left(\mathcal{U}\left(R_{j}\right), R_{j}, \eta_{m}\right)$

6: $\quad$ If $\mathcal{U}\left(R_{j}\right)$ finds another relay, then $R_{j}$ is available, otherwise it is not

7: end if

As for computational complexity, [20] shows that generalized fractional programming to solve (7) has polynomial complexity, whereas [5] shows that the complexity of the userrelay association procedure is quadratic in $K$ and $N$.

\section{NUMERICAL RESUlTS AND DisCUSSION}

A multi-user and relay-assisted network with $K=N=10$ is considered. Fast fading is modeled as zero-mean, unitvariance circularly symmetric Gaussian complex random variables, while the channel-to-noise ratios of all the user-relay and relay-destination channels are $u=10$ and $v=100$, respectively. Also, $P_{k, n}^{(c)}=0.1 \mathrm{~W}$ for all $k, \mu_{n}=\lambda_{k}=1$ for any $k$ and $n, B=1 \mathrm{MHz}$, and $q_{\max }=10 \mathrm{~W}$ for all $n$. Average results over $10^{4}$ system realizations are reported.

Figs. 2 and 3 show the minimum energy efficiency in (7a) versus $p_{\max }$ (assumed the same for all users), for DF and AF

${ }^{6}$ See [4] for further details about these functions. 


$$
\mathcal{H}_{g}\left(p_{k}, q_{n}\right)=\frac{1}{\left(p_{k}+q_{n}+1\right)^{2}}\left(\begin{array}{cc}
-\frac{\left(q_{n}^{2}+2 q_{n}+2 p_{k} q_{n}\right)}{\left(p_{k}+1\right)^{2}} & 1 \\
1 & -\frac{\left(p_{k}^{2}+2 p_{k}+2 p_{k} q_{n}\right)}{\left(q_{n}+1\right)^{2}}
\end{array}\right)
$$

relaying protocols, respectively. The following algorithms are compared: (a) the proposed joint power and relay allocation; (b) optimal power allocation by solving (7), with a random relay assignment; (c) optimal relay assignment by solving (10), with full power transmission. It is seen that the joint allocation of the transmit powers and relay assignment significantly outperforms the optimization of only the transmit powers or the relay assignments.

Finally, Tab. I shows the average number of outer iterations for Algorithm 1 to converge, versus $P_{\max }$. Convergence is declared when $\left|\min _{k} \mathrm{EE}_{k}^{(n)}-\min _{k} \mathrm{EE}_{k}^{(n-1)}\right| / \min _{k} \mathrm{EE}_{k}^{(n)} \leq$ $10^{-3}$, with $\min _{k} \mathrm{EE}_{k}^{(n)}$ denoting the minimum energy efficiency achieved in iteration $n$. The results indicate that convergence occurs in only a few iterations.

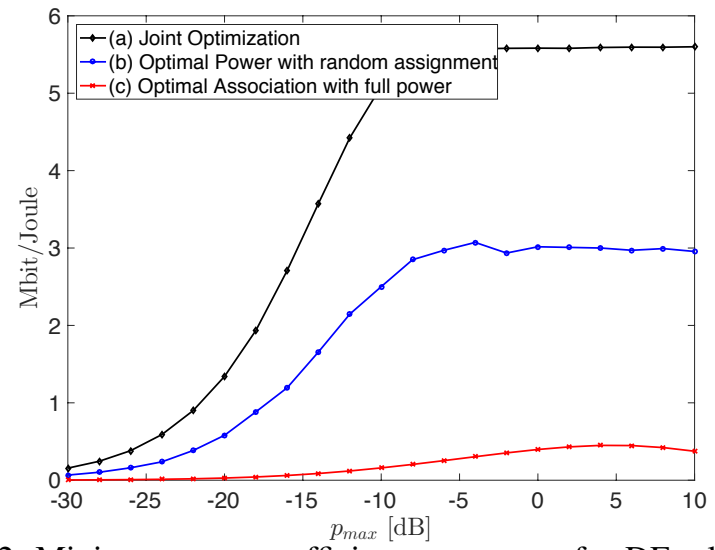

Fig. 2: Minimum energy efficiency vs. $p_{\max }$ for DF relaying.

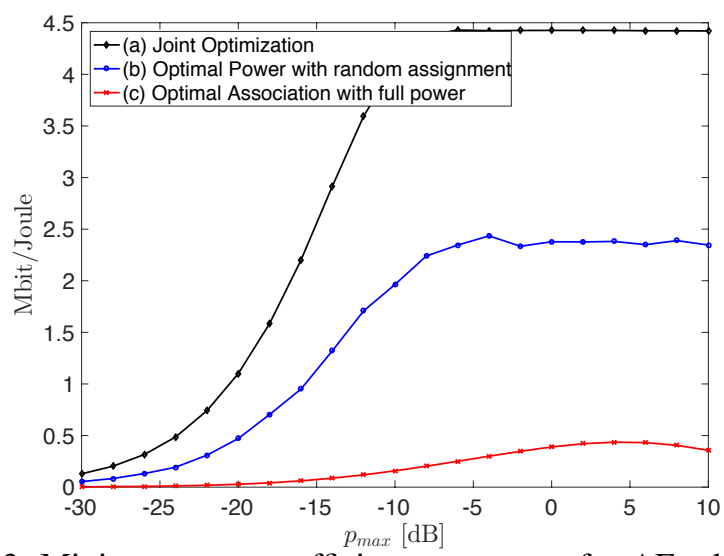

Fig. 3: Minimum energy efficiency vs. $p_{\max }$ for AF relaying.

\begin{tabular}{|c|c|c|c|c|c|}
\hline$P_{\max }[\mathrm{dB}]$ & -30 & -20 & -10 & 0 & 10 \\
\hline No. Iterations for AF & 2.82 & 2.89 & 3.71 & 3.85 & 3.94 \\
\hline No. Iterations for DF & 2 & 2.24 & 2.95 & 3.07 & 3.19 \\
\hline
\end{tabular}

TABLE I: Algorithm 1. Average iterations number vs. $P_{\max }$.

\section{CONCLUSION}

An algorithm for joint relay relay assignment and transmit power control has been developed to maximize the minimum among the users' energy efficiencies. The proposed approach merges alternating optimization and fractional programming theory, exhibiting a low complexity, while at the same time improving the energy efficiency compared to the optimization of only either the relay assignment or the transmit powers.

\section{REFERENCES}

[1] A. Zappone and E. Jorswieck, "Energy efficiency in wireless networks via fractional programming theory," Foundations and Trends in Commun. and Inform. Theory, vol. 11, no. 3-4, pp. 185-396, 2015.

[2] L. Sanguinetti, A. A. D'Amico, and Y. Rong, "A tutorial on the optimization of amplify-and-forward MIMO relay systems," IEEE $J$. Sel. Areas Commun., vol. 30, no. 8, pp. 1331-1346, Sept. 2012.

[3] A. Bletsas, A. Khisti, D. P. Reed, and A. Lippman, "A simple cooperative diversity method based on network path selection," IEEE J. Select. Areas Commun., vol. 24, no. 3, pp. 659-672, Mar. 2006.

[4] S. Sharma, Y. Shi, Y. T. Hou, and S. Kompella, "An optimal algorithm for relay node assignment in cooperative ad hoc networks," IEEE/ACM Trans. Networking, vol. 19, no. 3, pp. 879-892, June 2010.

[5] S. Atapattu, Y. Jing, H. Jiang, and C. Tellambura, "Relay selection and performance analysis in multiple-user networks," IEEE J. Select. Areas Commun., vol. 31, no. 8, pp. 1517-1529, Aug. 2013.

[6] M. Zhou, Q. Cui, R. Jantti, and X. Tao, "Energy-efficient relay selection and power allocation for two-way relay channel with analog network coding," IEEE Commun. Lett., vol. 16, no. 6, pp. 816-819, Jun. 2012.

[7] G. d. S. Peron, G. Brante, and R. D. Souza, "Energy-efficient distributed power allocation with multiple relays and antenna selection," IEEE Trans. Commun., vol. 63, no. 12, pp. 4797-4808, Dec. 2015.

[8] J. Kim, K. Kim, and J. Lee, "Energy-efficient relay selection of cooperative HARQ based on the number of transmissions over rayleigh fading channels," IEEE Trans. Veh. Technol., vol. 66, no. 1, pp. 610-621, 2017.

[9] C. Li, F. Sun, and J. M. Cioffi, "Energy efficient MIMO relay transmissions via joint power allocations," IEEE Trans. on Circuits and Syst. II, vol. 61, no. 7, pp. 531-535, July 2014.

[10] C. Li, H. J. Yang, F. Sun, J. M. Cioffi, and L. Yang, "Approximate closed-form energy efficient PA for MIMO relaying systems in the high SNR regime," IEEE Commun. Lett., vol. 18, no. 8, pp. 1367-1370, June 2014.

[11] Z. Sheng et al., "Energy-efficient relay selection for cooperative relaying in wireless multimedia networks," IEEE Trans. Veh. Technol., vol. 64, no. 3, pp. 1156-1170, Mar. 2015.

[12] A. Zappone, E. A. Jorswieck, and S. Buzzi, "Energy efficiency and interference neutralization in two-hop MIMO interference channels," IEEE Trans. Signal Process., vol. 62, no. 24, pp. 6481-6495, Dec. 2014

[13] C. Luo et al., "Energy-efficient distributed relay and power control in cognitive radio cooperative communications," IEEE J. Sel. Areas Commun., vol. 31, no. 11, pp. 2442-2452, Nov. 2013.

[14] D. Gündüz et al., "The multiway relay channel," IEEE Trans. Inform. Theory, vol. 59, no. 1, pp. 51-63, Jan. 2013.

[15] B. Matthiesen, A. Zappone, and E. A. Jorswieck, "Resource allocation for energy-efficient 3-way relay channels," IEEE Trans. on Wireless Commun., vol. 14, no. 8, pp. 4454-4468, Aug. 2015.

[16] J. Yang et al., "Centralized energy-efficient multiuser multiantenna relaying in next-generation radio access networks," IEEE Trans. Veh. Technol., vol. 66, no. 9, pp. 7913-7924, Sep. 2017.

[17] Y. Chen, X. Fang, and B. Huang, "Energy-efficient relay selection and resource allocation in nonregenerative relay OFDMA systems," IEEE Trans. Veh. Technol., vol. 63, no. 8, pp. 3689-3699, Oct. 2014.

[18] S. Atapattu, Y. Jing, H. Jiang, and C. Tellambura, "Relay selection schemes and performance analysis approximations for two-way networks," IEEE Trans. Commun., vol. 61, no. 3, pp. 987-998, Mar. 2013.

[19] Y. Feng, Z. Yang, S. Yan, N. Yang, and B. Lv, "Physical layer security enhancement in multi-user multi-full-duplex-relay networks," in IEEE International Communication Conference (ICC), 2017.

[20] J.-P. Crouzeix and J. A. Ferland, "Algorithms for generalized fractional programming," Math. Prog., vol. 52, no. 1-3, pp. 191-207, 1991. 